\title{
ON GENERALIZED QUASI-EINSTEIN GRW SPACE-TIMES
}

\author{
UDAY CHAND DE AND SAMEH SHENAWY
}

\begin{abstract}
Recently, it is proven that generalized Robertson-Walker spacetimes in all orthogonal subspaces of Gray's decomposition but one(unrestricted) are perfect fluid space-times. GRW space-times in the unrestricted subspace are identified by having constant scalar curvature. Generalized quasi-Einstein GRW space-times have a constant scalar curvature. It is shown that generalized quasi-Einstein GRW space-times reduce to Einstein space-times or perfect fluid space-times.
\end{abstract}

\section{INTRODUCTION}

The warped product $M=I \times{ }_{f} M^{*}$ of an open connected interval $\left(I,-\mathrm{d} t^{2}\right)$ of $\mathbb{R}$ and a Riemannian manifold $M^{*}$ with warping function $f: I \rightarrow \mathbb{R}^{+}$is called a generalized Robertson-Walker space-time(or GRW space-times) [12, 15]. This family of Lorentzian space-times broadly extends the classical Robertson-Walker space-times, Friedmann cosmological models, Einstein-de Sitter space-times and many others [2,15. The classical Robertson-Walker spacetime is regarded as cosmological models since it is spatially homogeneous and spatially isotropic whereas GRW space-times serve as inhomogeneous extension of Robertson-Walker spacetimes that admit an isotropic radiation [2] (see also [4,15]). A Lorentzian manifold is called a perfect fluid space-time if the Ricci tensor Ric takes the form

$$
\operatorname{Ric}(X, Y)=a g(X, Y)+b A(X) A(Y)
$$

where $a, b$ are scalars and $A$ is a 1 -form metrically equivalent to a unit time-like vector field 1314. Perfect fluid space-times in the language of differential geometry are called quasi-Einstein spaces where $A$ is metrically equivalent to a unit spacelike vector field. Recently, in [14, it is proven that a perfect fluid space-time with divergence-free conformal curvature tensor is a GRW space-time with Einstein fibers given that the scalar curvature is constant. Many sufficient conditions on perfect fluid space-times to be a GRW space-time are derived.

Gray presented an invariant orthogonal decomposition of the covariant derivative of the Ricci tensor [5] (see also [10]). Recently, Carlo Mantica et al proved that the Ricci tensor of a generalized Robertson-Walker space-time in all classes of Gray's decomposition but $\mathcal{A} \oplus \mathcal{B}$ is either Einstein or takes the form of a perfect fluid whereas $\mathcal{A} \oplus \mathcal{B}$ is not restricted [13]. The class $\mathcal{A} \oplus \mathcal{B}$ is characterized by $\nabla R=0$ i.e. the scalar curvature is constant. Now, the following question arises.

Does the Ricci tensor of all GRW space-times in $\mathcal{A} \oplus \mathcal{B}$ reduce to be Einstein or take the form of a perfect fluid?

2000 Mathematics Subject Classification. Primary 53C25; Secondary 83F05.

Key words and phrases. Quasi-Einstein manifolds, Perfect fluid space-times, Generalized Robertson-Walker space-times, Einstein manifolds. 
In this work, we get a partial positive answer. A (pseudo-)Riemannian manifold $(M, g)$ is called a generalized quasi-Einstein manifold if its Ricci curvature satisfies

$$
\operatorname{Ric}(X, Y)=\alpha g(X, Y)+\beta A(X) A(Y)+\gamma[A(X) B(Y)+A(Y) B(X)],
$$

where $\alpha, \beta$ and $\gamma$ are non-zero constants, $A$ and $B$ are 1 -forms corresponding to two orthonormal vector field [1,3,6, 9. If $\gamma=0$, then $M$ reduces to a quasi-Einstein manifold. It is clear that generalized quasi-Einstein space-times are generally imperfect fluid space-times with constant scalar curvature $R=n \alpha+\beta$. However, we prove that generalized quasi-Einstein GRW space-times are either Einstein or perfect fluid space-times.

Remark 1. It is noted that any vector field orthogonal to a time-like vector field is space-like. Thus the generators couldn't be time-like. Now, one may assume that one of the generators is time-like and the other is space-like. Generally, the results of this article still hold in this case with minor changes.

\section{Notes on GENERALIZED QUASI-EINSTEIN MANIFOLDS}

Let $M$ be a generalized quasi-Einstein (pseudo-)Riemannian manifold i.e.

$$
R_{i j}=\alpha g_{i j}+\beta A_{i} A_{j}+\gamma\left(A_{i} B_{j}+A_{j} B_{i}\right),
$$

where $A_{i} A^{i}=B_{j} B^{j}=1$ and $A_{i} B^{i}=0$. The trace of this equations gives

$$
R=n \alpha+\beta \text {. }
$$

It is noted that

$$
\begin{aligned}
A^{i} A^{j} R_{i j} & =\alpha+\beta, \\
A^{i} B^{j} R_{i j} & =\gamma, \\
B^{i} B^{j} R_{i j} & =\alpha,
\end{aligned}
$$

and consequently $\beta=\left(A^{i} A^{j}-B^{i} B^{j}\right) R_{i j}$. Hence we can state the following result.

Proposition 1. Let $M$ be a generalized quasi-Einstein manifold with generators $A$ and $B$. Then the scalar curvature is

$$
R=(n-1) B^{i} B^{j} R_{i j}+A^{i} A^{j} R_{i j}
$$

Now assume that $A$ is an eigenvector of the Ricci tensor with eigenvalue $\xi$ i.e. $A^{i} R_{i j}=\xi A_{j}$. A contraction of Equation (2.1) with $A^{i}$ yields

$$
A^{i} R_{i j}=\alpha A^{i} g_{i j}+\beta A^{i} A_{i} A_{j}+\gamma\left(A^{i} A_{i} B_{j}+A^{i} A_{j} B_{i}\right)
$$

implies $(\xi-\alpha-\beta) A_{j}=\gamma B_{j}$. Thus $\gamma=0$ and $\xi=\alpha+\beta$. If $B$ is an eigenvector of the Ricci tensor with eigenvalue $\phi$, then

$$
B^{i} R_{i j}=\alpha B^{i} g_{i j}+\beta B^{i} A_{i} A_{j}+\gamma\left(B^{i} A_{i} B_{j}+B^{i} A_{j} B_{i}\right)
$$

infers

$$
(\phi-\alpha) B_{j}=\gamma A_{j}
$$

Consequently, $\phi=\alpha$ and $\gamma=0$. Conversely, assume that $M$ is a quasi-Einstein manifold with generator $A$. Then

$$
R_{i j}=\alpha g_{i j}+\beta A_{i} A_{j}
$$

yields

$$
A^{i} R_{i j}=\alpha A^{i} g_{i j}+\beta A^{i} A_{i} A_{j}=(\alpha+\beta) A_{j}
$$


Also,

$$
B^{i} R_{i j}=\alpha B^{i} g_{i j}+\beta B^{i} A_{i} A_{j}=\alpha A_{j} .
$$

This leads to the following.

Theorem 1. Let $M$ be a generalized quasi-Einstein manifold. Then, $M$ reduces to a quasi-Einstein manifold if and only if one of the generators is an eigenvector of the Ricci tensor.

The covariant derivative of the Ricci tensor of a generalized quasi-Einstein manifold is given by

$$
\begin{aligned}
\nabla_{k} R_{i j}= & \beta\left(\nabla_{k} A_{i}\right) A_{j}+\beta A_{i}\left(\nabla_{k} A_{j}\right) \\
& +\gamma\left[\left(\nabla_{k} A_{i}\right) B_{j}+A_{i}\left(\nabla_{k} B_{j}\right)+\left(\nabla_{k} A_{j}\right) B_{i}+A_{j}\left(\nabla_{k} B_{i}\right)\right]
\end{aligned}
$$

and hence

$$
\begin{aligned}
\nabla_{i} R_{k j}= & \beta\left(\nabla_{i} A_{k}\right) A_{j}+\beta A_{k}\left(\nabla_{i} A_{j}\right) \\
& +\gamma\left[\left(\nabla_{i} A_{k}\right) B_{j}+A_{k}\left(\nabla_{i} B_{j}\right)+\left(\nabla_{i} A_{j}\right) B_{k}+A_{j}\left(\nabla_{i} B_{k}\right)\right]
\end{aligned}
$$

Thus, the Codazzi deviation tensor $\mathcal{D}$ is

$$
\begin{aligned}
\mathcal{D}_{k i j}= & \nabla_{k} R_{i j}-\nabla_{i} R_{k j} \\
= & \beta\left(\nabla_{k} A_{i}\right) A_{j}+\beta A_{i}\left(\nabla_{k} A_{j}\right)-\beta\left(\nabla_{i} A_{k}\right) A_{j}-\beta A_{k}\left(\nabla_{i} A_{j}\right) \\
& +\gamma\left[\left(\nabla_{k} A_{i}\right) B_{j}+A_{i}\left(\nabla_{k} B_{j}\right)+\left(\nabla_{k} A_{j}\right) B_{i}+A_{j}\left(\nabla_{k} B_{i}\right)\right] \\
& -\gamma\left[\left(\nabla_{i} A_{k}\right) B_{j}+A_{k}\left(\nabla_{i} B_{j}\right)+\left(\nabla_{i} A_{j}\right) B_{k}+A_{j}\left(\nabla_{i} B_{k}\right)\right]
\end{aligned}
$$

Now, we have the following cases

$$
\begin{aligned}
A^{j} \mathcal{D}_{k i j} & =\beta\left(\nabla_{k} A_{i}-\nabla_{i} A_{k}\right)+\gamma\left(\nabla_{k} B_{i}-\gamma \nabla_{i} B_{k}\right) \\
B^{j} \mathcal{D}_{k i j} & =\nabla_{k} A_{i}-\nabla_{i} A_{k}
\end{aligned}
$$

Thus, for a Codazzi Ricci tensor, the generators are both closed. In this case,

$$
\begin{aligned}
0= & \mathcal{D}_{k i j} \\
= & \beta A_{i}\left(\nabla_{k} A_{j}\right)-\beta A_{k}\left(\nabla_{i} A_{j}\right)+\gamma A_{i}\left(\nabla_{k} B_{j}\right) \\
& +\gamma\left(\nabla_{k} A_{j}\right) B_{i}-\gamma A_{k}\left(\nabla_{i} B_{j}\right)-\gamma\left(\nabla_{i} A_{j}\right) B_{k} \\
= & \nabla_{j}\left[\beta A_{i} A_{k}+\gamma A_{i} B_{k}+\gamma A_{k} B_{i}\right] \\
& -2 \beta A_{k}\left(\nabla_{j} A_{i}\right)-2 \gamma\left(\nabla_{j} A_{i}\right) B_{k}-2 \gamma A_{k}\left(\nabla_{j} B_{i}\right) \\
= & \nabla_{j} R_{i k}-2\left(\beta A_{k}+\gamma B_{k}\right) \nabla_{j} A_{i}-2 \gamma A_{k} \nabla_{j} B_{i} .
\end{aligned}
$$

Thus we have the following.

Proposition 2. Let $M$ be a generalized quasi-Einstein manifold. Assume that $M$ is Einstein-like of class $\mathcal{B}$ (i.e. the Ricci tensor is a Codazzi tensor). Then $A$ and $B$ are closed. Moreover,

$$
\nabla_{j} R_{i k}=2\left(\beta A_{k}+\gamma B_{k}\right) \nabla_{j} A_{i}+2 \gamma A_{k} \nabla_{j} B_{i} .
$$

A contraction of $\mathcal{D}_{k i j}$ by $g^{i j}$ and then by the generators $A^{k}$ and $B^{k}$ infers

$$
\begin{aligned}
& 0=\left(\beta A^{i}+\gamma B^{i}\right) \nabla_{i} A_{k}+\left(\beta A_{k}+\gamma B_{k}\right)\left(\nabla_{i} A^{i}\right)+\gamma A_{k}\left(\nabla_{i} B^{i}\right)+\gamma A^{i} \nabla_{i} B_{k}, \\
& 0=\beta\left(\nabla_{i} A^{i}\right)+\gamma \nabla_{i} B^{i}, \\
& 0=\gamma \nabla_{i} A^{i} .
\end{aligned}
$$

Thus $\nabla_{i} A^{i}=\nabla_{i} B^{i}=0$. 
Assume that $M$ is Einstein-like of class $\mathcal{P}$ ( that is, the Ricci tensor is a parallel, $\left.\nabla_{k} R_{i j}=0\right)$. Then,

$$
\begin{aligned}
0= & \beta\left(\nabla_{i} A_{k}\right) A_{j}+\beta A_{k}\left(\nabla_{i} A_{j}\right)+\gamma\left(\nabla_{i} A_{k}\right) B_{j} \\
& +\gamma A_{k}\left(\nabla_{i} B_{j}\right)+\gamma\left(\nabla_{i} A_{j}\right) B_{k}+\gamma A_{j}\left(\nabla_{i} B_{k}\right)
\end{aligned}
$$

Contractions by $A^{k}$ and $B^{k}$ imply

$$
\begin{aligned}
& 0=\beta \nabla_{i} A_{j}+\gamma \nabla_{i} B_{j}, \\
& 0=\gamma \nabla_{i} A_{j} .
\end{aligned}
$$

Assume that $A$ is not parallel, then $\beta=\gamma=0$. Thus we conclude.

Theorem 2. Let $M$ be a Ricci-symmetric generalized quasi-Einstein manifold. Then, $M$ is Einstein if the generator $A$ is not covariantly constant.

\section{Generalized QuAsi-Einstein GRW SPACE-TimeS}

A Lorentzian manifold $M$ is a GRW space-time if and only if $M$ has a unit time-like vector field $u_{i}$ such that

$$
\nabla_{k} u_{j}=\varphi\left(g_{k j}+u_{k} u_{j}\right)
$$

which is also an eigenvector of the Ricci tensor i.e. $R_{i j} u^{i}=\xi u_{j}$ for some scalar functions $\varphi$ and $\xi[11-13$. We say that $u$ is a nontrivial torse-forming vector field if $\varphi \neq 0$. This characterization is an alternative of Chen's theorem in [2]. If $M$ is a generalized quasi-Einstein manifold, then

$$
R_{i j}=\alpha g_{i j}+\beta A_{i} A_{j}+\gamma\left(A_{i} B_{j}+A_{j} B_{i}\right) .
$$

A contraction by $u^{i}$ yields

$$
u^{i} R_{i j}=\alpha u^{i} g_{i j}+\beta u^{i} A_{i} A_{j}+\gamma\left(u^{i} A_{i} B_{j}+A_{j} u^{i} B_{i}\right)
$$

which implies

$$
(\xi-\alpha) u_{j}=\beta\left(u^{i} A_{i}\right) A_{j}+\gamma\left(u^{i} A_{i}\right) B_{j}+\gamma A_{j}\left(u^{i} B_{i}\right)
$$

and hence

$$
(\xi-\alpha) u_{j}=\left[\beta\left(u^{i} A_{i}\right)+\gamma\left(u^{i} B_{i}\right)\right] A_{j}+\gamma\left(u^{i} A_{i}\right) B_{j}
$$

Two different contractions by the generators give

$$
(\xi-\alpha-\beta)\left(u^{i} A_{i}\right)-\gamma\left(u^{i} B_{i}\right)=0
$$

and

$$
(\xi-\alpha)\left(u^{i} B_{i}\right)-\gamma\left(u^{i} A_{i}\right)=0
$$

Thus

$$
\begin{aligned}
(\xi-\alpha) u_{j} & =\left[\beta\left(u^{i} A_{i}\right)+(\xi-\alpha-\beta)\left(u^{i} A_{i}\right)\right] A_{j}+(\xi-\alpha)\left(u^{i} B_{i}\right) B_{j} \\
& =(\xi-\alpha)\left(u^{i} A_{i}\right) A_{j}+(\xi-\alpha)\left(u^{i} B_{i}\right) B_{j}
\end{aligned}
$$

and hence

$$
(\xi-\alpha)\left[u_{j}-\left(u^{i} A_{i}\right) A_{j}-\left(u^{i} B_{i}\right) B_{j}\right]=0 .
$$

It is clear that $u_{j}$ is not a linear combination of $A_{j}$ and $B_{j}$ only since $u^{i}$ is time-like whereas $A^{i}$ and $B^{i}$ are orthonormal space-like fields so $\xi=\alpha$. Therefore,

$$
\begin{aligned}
\beta\left(u^{i} A_{i}\right)+\gamma\left(u^{i} B_{i}\right) & =0 \\
\gamma\left(u^{i} A_{i}\right) & =0
\end{aligned}
$$


It is noted that $\gamma=\beta=0$ if $\left(u^{i} A_{i}\right)$ is not zero. Suppose that $\left(u^{i} B_{i}\right)$ does not vanish. Then Equation (3.3) implies that either $\gamma=0$ or $\left(u^{i} A_{i}\right)=0$. The later case with Equation (3.2) yield $\gamma=0$ i.e. $M$ is quasi-Einstein if $\left(u^{i} B_{i}\right) \neq 0$. Now, assume that $u^{i}$ is orthogonal to both the generators i.e. $\left(u^{i} A_{i}\right)=\left(u^{i} B_{i}\right)=0$. The Ricci tensor of a GQE manifold is

$$
R_{i j}=\xi g_{i j}+\beta A_{i} A_{j}+\gamma\left(A_{i} B_{j}+A_{j} B_{i}\right)
$$

and so

$$
\nabla_{k} R_{i j}=\beta A_{j} \nabla_{k} A_{i}+\beta A_{i} \nabla_{k} A_{j}+\gamma\left(B_{j} \nabla_{k} A_{i}+A_{i} \nabla_{k} B_{j}+A_{j} \nabla_{k} B_{i}+B_{i} \nabla_{k} A_{j}\right)
$$

A contraction by $u^{i}$ implies

$$
\begin{aligned}
u^{i} \nabla_{k} R_{i j} & =0 \\
\nabla_{k}\left(u^{i} R_{i j}\right)-R_{i j} \nabla_{k} u^{i} & =0
\end{aligned}
$$

It is noted that $u^{i}$ is an eigenvector of the Ricci tensor ( i.e. $u^{i} R_{i j}=\xi u_{j}$ ) and $\nabla_{k} u^{i}=\varphi\left(\delta_{k}^{i}+u_{k} u^{i}\right)$. Thus

$$
\begin{aligned}
\nabla_{k}\left(\xi u_{j}\right)-R_{i j} \varphi\left(\delta_{k}^{i}+u_{k} u^{i}\right) & =0 \\
\xi \nabla_{k} u_{j}-\varphi \delta_{k}^{i} R_{i j}-\varphi u_{k} u^{i} R_{i j} & =0 \\
\xi \varphi\left(g_{k j}+u_{k} u_{j}\right)-\varphi R_{k j}-\varphi \xi u_{k} u_{j} & =0 \\
\varphi\left(\xi g_{k j}-R_{k j}\right) & =0
\end{aligned}
$$

So $M$ is Einstein if $u$ is a nontrivial torse-forming vector field.

Theorem 3. Let $M$ be a generalized quasi-Einstein GRW space-time. Then $u^{i} R_{i j}=$ $\alpha u_{j}$ i.e. $\alpha$ is the eigenvalue of the eigenvector $u^{i}$ and

(1) $M$ reduces to be Einstein space-time if $u^{i}$ is orthogonal to both the generators provided $\varphi \neq 0$.

(2) $M$ reduces to be Einstein space-time if $u^{i}$ is not orthogonal to first generator.

(3) $M$ reduces to be perfect fluid space-time if $u^{i}$ is not orthogonal to the second generator.

Corollary 1. Let $M$ be a generalized quasi-Einstein Lorentzian manifold admitting a unit time-like non-trivial torse-forming vector field. Then $M$ reduces to an Einstein GRW space-time or a perfect fluid GRW space-time.

\section{REFERENCES}

[1] Chaki, M. C. On generalized quasi Einstein manifolds, Publ. Math. Debrecen 58 (2001): 683-691.

[2] Chen, Bang-Yen. A simple characterization of generalized Robertson-Walker spacetimes, General Relativity and Gravitation 46, no. 12 (2014): 1833.

[3] De, Avik, Ahmet Yildiz, and Uday Chand De. On Generalized Quasi Einstein Manifolds, Filomat 28, no. 4 (2014): 811-820.

[4] Ehlers, Jurgen, P. Geren, and Rainer K. Sachs. "Isotropic Solutions of the Einstein-Liouville Equations." Journal of Mathematical Physics 9, no. 9 (1968): 1344-1349.

[5] Gray, Alfred. "Einstein-like manifolds which are not Einstein. Geometriae dedicata 7, no. 3 (1978): 259-280.

[6] Guler, Sinem, and Sezgin Altay Demirbag. On Ricci symmetric generalized quasi Einstein spacetimes, Miskolc Mathematical Notes 16, no. 2 (2015): 853-868.

[7] Guler, Sinem, and Sezgin Altay Demirbag. A Study of Generalized Quasi Einstein Spacetimes with Applications in General Relativity, International Journal of Theoretical Physics 55, no. 1 (2016): 548-562. 
[8] Guler, Sinem, and Sezgin Altay Demirbag. Riemannian Manifolds Satisfying Certain Conditions on Pseudo-Projective Curvature Tensor, Filomat 30, no. 3 (2016): 721-731.

[9] Mallick, Sahanous, and Uday Chand De. On a Class of Generalized quasi-Einstein Manifolds with Applications to Relativity, Acta Universitatis Palackianae Olomucensis. Facultas Rerum Naturalium. Mathematica 55, no. 2 (2016): 111-127.

[10] Mantica, Carlo Alberto, and Luca Guido Molinari. Riemann compatible tensors, In Colloquium Mathematicum, vol. 128, pp. 197-210. Instytut Matematyczny Polskiej Akademii Nauk, 2012.

[11] Mantica, Carlo Alberto, and Luca Guido Molinari. On the Weyl and Ricci tensors of Generalized Robertson-Walker space-times, Journal of Mathematical Physics 57, no. 10 (2016): 102502.

[12] Mantica, Carlo Alberto, and Luca Guido Molinari. Generalized Robertson-Walker spacetimes - a survey, International Journal of Geometric Methods in Modern Physics 14, no. 03 (2017): 1730001.

[13] Mantica, Carlo Alberto, Luca Guido Molinari, Young Jin Suh, and Sameh Shenawy. PerfectFluid, Generalized Robertson-Walker Space-times, and Gray's Decomposition, Journal of Mathematical Physics, revised.

[14] Mantica, Carlo Alberto, Uday Chand De, Young Jin Suh, and Luca Guido Molinari. " Perfect fluid spacetimes with harmonic generalized curvature tensor." Osaka Journal of Mathematics 56, no. 1 (2019): 173-182.

[15] Sanchez, Miguel. "On the geometry of generalized Robertson-Walker spacetimes: geodesics." General Relativity and Gravitation 30, no. 6 (1998): 915-932.

(U. C. De) Department of Pure Mathematics, University of Calcutta, 35, Ballygaunge Circular Road, Kolkata 700019, West Bengal, India,

E-mail address: uc_de@yahoo.com

(S. Shenawy) Basic Science Department, Modern Academy for Engineering and TechNOLOGY, MAADI, EGYPT,

E-mail address: drshenawy@mail.com 